\title{
Respiratory infection following tracheostomy
}

\author{
M. S. GOTSMAN AND J.L. WHITBY \\ From the Queen Elizabeth Hospital, Edgbaston, Birmingham. 15
}

Tracheostomy has been practised for centuries for the relief of respiratory tract obstruction. Its use has increased very markedly since the introduction of intermittent positive pressure respiration (I.P.P.R.) in the treatment of poliomyelitis (Galloway, 1943; Lassen, 1953). Its value has since been proved for other conditions associated with respiratory paralysis, such as tetanus (Cole, 1959 ; Smythe and Bull, 1961) and myasthenia gravis. It is also used in respiratory failure characterized by secretional ventilatory obstruction (Nelson, 1958), as is found in severe head injuries (Bryce-Smith, 1950 ; Maciver, Frew, and Matheson, 1958; Cawthorne, Hewlett, and Ranger, 1959) or in chronic bronchitis (HughJones, 1958 ; Davidson, 1959) or in unconscious patients who are unable to cough up retained secretions (Nelson, 1958).

These authors and numerous others (Atkins, 1960 ; Meade, 1961 ; Head, 1961) are enthusiastic advocates of tracheostomy, and they stress the urgency of performing the operation when indicated and the undoubted benefits obtained from its use. Little attention has been paid to the post-operative respiratory sequelae. We have been impressed by the frequency with which these patients have become infected with hospital crossinfecting organisms, particularly staphylococci. They are very susceptible to infection and, when infected, form a reservoir dangerous to other patients.

We have traced all cases of tracheostomy performed in the Queen Elizabeth Hospital in 1961 in order to assess the incidence and significance of infection following tracheostomy.

\section{METHODS}

Clinical material Patients on whom tracheostomy had been performed in 1961 were identified from the operating theatre records for that year. Tracheostomy is normally performed in an operating theatre by a surgical registrar, but any tracheostomy performed in the wards would not be recorded and could not be included in this survey. Patients whose clinical records were inadequate for assessment were also excluded. Four additional selected patients had a tracheostomy performed in 1962 and have been used for illustrative but not statistical purposes.

BACTERIOLOGICAL MATERIAL This was assessed from the patients' notes, laboratory records, and any epidemiological data available. The phage types of all staphylococci isolated from patients were included and examined in relation to staphylococci isolated throughout the hospital. This was possible as all staphylococci are phage typed as a routine.

NECROPSY MATERIAL Full reports and histological preparations were examined from all patients who had been submitted to necropsy. An attempt was made to correlate these findings with the incidence of postoperative infection.

\section{RESULTS OF THE STUDY}

INDICATIONS FOR TRACHEOSTOMY Forty patients underwent tracheostomy in the operating theatre but only 29 of these could be included in the survey. For the remaining 11 patients the data available were inadequate for an assessment to be made.

The indications for which tracheostomy was performed are listed in Table $I$ and illustrated in Figure 1.

\section{TABLE I} INDICATION FOR TRACHEOSTOMY

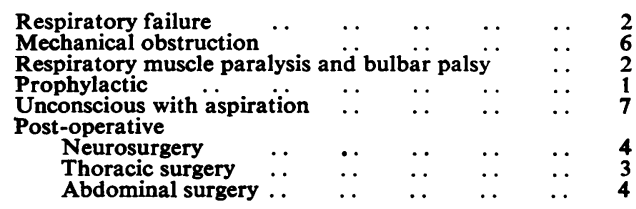

Six patients required tracheostomy for mechanical obstruction of the upper respiratory tract, and in two of these patients the obstruction was precipitated by direct endoscopy.

Secretional ventilatory obstruction in one form or another was the indication for tracheostomy in all the remaining patients. These patients were either unwilling or unable to cough up retained secretions.

Prolonged unconsciousness and an inability to cough up the retained secretions accounted for 11 
patients. These have been listed under two headings. In one group unconsciousness persisted or occurred following a neurosurgical procedure. In the other group, neurological conditions were often present (head injuries and cerebrovascular accidents) but neurosurgery was not undertaken.

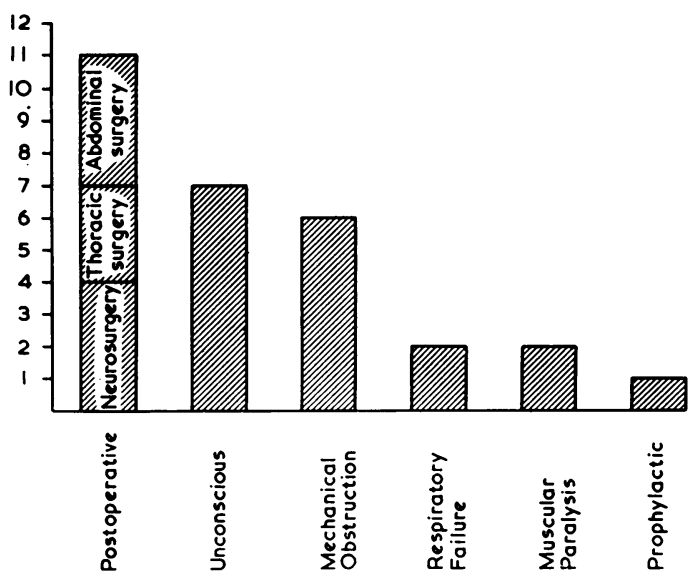

FIG. 1. Indications for tracheostomy.

Four patients on whom tracheostomy was performed after upper abdominal surgery had a stormy post-operative course. One had severe chronic bronchitis and the others had prolonged ileus and required additional operations. During this period of poor consciousness they gave the nursing staff little co-operation with cough and expectoration and were hampered by abdominal distension and pain. Accumulated sputum could not be removed and respiratory insufficiency occurred.

The patients who required tracheostomy after thoracic surgery all had pre-operative respiratory insufficiency and failed to tolerate the additional strain of the operation.

Two patients had severe chronic bronchitis and intractable respiratory failure.

Respiratory muscle paralysis and bulbar palsy accounted for a further two patients. One patient had polyneuritis and the other received muscular relaxants to relieve tetanic spasms. Intermittent positive pressure respiration was applied through the tracheostomy. There were no cases of poliomyelitis.

One patient, an elderly lady who had chronic bronchitis and who required cholecystectomy, had a prophylactic tracheostomy. This was successful and she was one of the few who did not develop a subsequent respiratory infection.

INCIDENCE OF RESPIRATORY INFECTION AFTER TRACHEOSTOMY We have regarded respiratory infection to be acute bronchitis or tracheitis or $\underset{\vec{\rho}}{\vec{\rho}}$ bronchopneumonia. The criteria used in assessing infection were clinical; fever, tachypnoea, $\frac{}{\partial}$ purulent sputum, clinical signs in the chest, and radiographic changes. Infection was never $\widehat{\nabla}$ diagnosed solely on the basis of bacteriological reports. These were used only to designate the causative organisms when the criteria of infection $\overrightarrow{0}$ had been established.

Six patients survived for less than three days $\vec{\omega}$ after tracheostomy. This period was too short to $\rightleftharpoons$ assess evidence of post-operative infection.

Of the remaining 23 patients, 18 developed $\overrightarrow{0}$ infection. It is important to determine as far as possible whether such infection was newlyc acquired or reactivation of existing foci. Sputum 음 was not examined pre-operatively in some patients, but, when it was, notably in the chronic bronchitics, it was found that the post-operative infections were due to organisms different from those isolated previously.

The data were ther examined for any $\overrightarrow{\mathscr{D}}$ predisposing causes.

1. Previous chronic chest disease The series of patients is too small to draw any conclusion about the susceptibility of bronchitic patients to infection after tracheostomy (Table II).

TABLE I I

PREVIOUS CHRONIC CHEST PATHOLOGY AND SUB-을 SEQUENT INFECTION

\begin{tabular}{l|c|c|c|c}
\hline & $\begin{array}{c}\text { Total } \\
\text { No. }\end{array}$ & Infected & $\begin{array}{c}\text { Not } \\
\text { Infected }\end{array}$ & $\begin{array}{c}\text { Too Short } \\
\text { a Time to } \\
\text { Assess }\end{array}$ \\
\hline $\begin{array}{l}\text { Previous chronic bronchitis } \\
\text { Kyphoscoliosis . }\end{array}$ & 10 & 5 & 3 & 2 \\
$\begin{array}{c}\text { No history of previous } \\
\text { chronic chest pathology }\end{array}$ & 18 & 12 & 2 & 4 \\
\hline
\end{tabular}

2. Acute respiratory infection before tracheostomy In addition to chronic bronchitis other

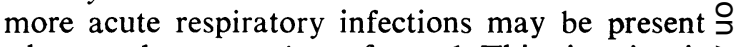
when tracheostomy is performed. This situation is $\frac{D}{O}$ examined in Table III and illustrated in Figure 2.

TA B LE III

INFECTION IN THE RESPIRATORY TRACT PRIOR TO N TRACHEOSTOMY AND ITS RELATION TO SUBSEQUENT O TRACHEOSTOMY AND INFECTION

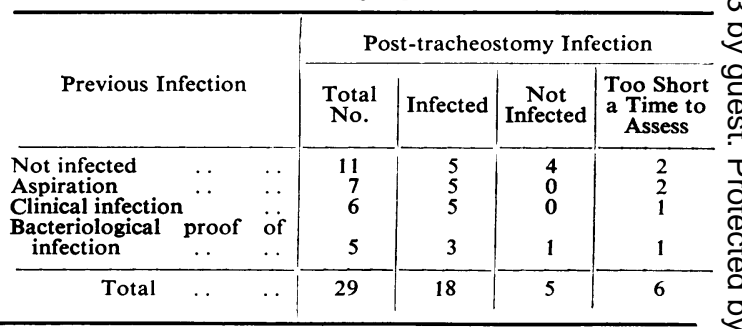




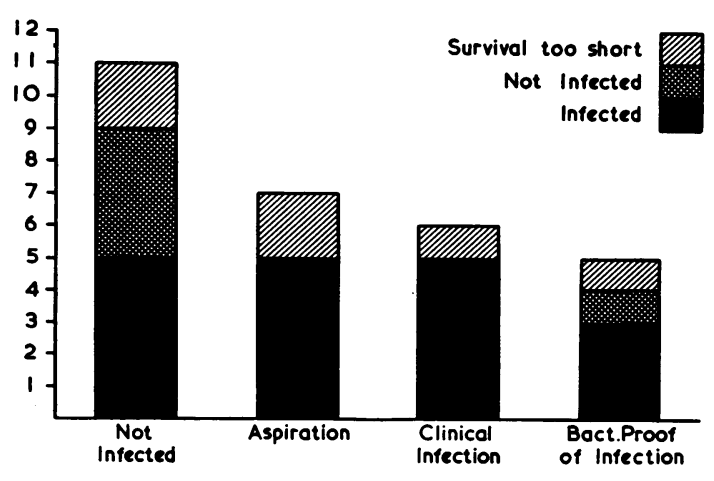

FIG. 2. Pre-existing chest infection in relation to posttracheostomy infection.

The patients were divided into four groups. Five had overt infection with positive bacteriological cultures from the sputa; those showing infection after tracheostomy were infected with different organisms. In six patients there was clinical evidence of respiratory infection but bacteriological data were not obtained. Seven patients are considered to have aspirated vomitus or inhaled sputum during a period of unconsciousness. Aspiration in an unconscious patient must lead to a pneumonia. Eleven patients had no evidence of any pre-existing infection.

Where evidence of previous infection existed, it was difficult to assess what role the tracheostomy had played in any subsequent infection. The nature of the infecting organisms in most cases suggests the occurrence of fresh infection, and this point is further considered later.

3. Intermittent positive pressure respiration This was required in eight patients. Seven of them developed respiratory tract infection.

4. Effect of previous antibiotic therapy Antibiotic administration was examined in the 23 patients who survived long enough for evidence of post-operative infection to be assessed. Table IV shows the relation of therapy to subsequent infection. Prophylactic administration of antibiotics in the absence of infection, in the small group which

TAB L E I V

ANTIBIOTIC ADMINISTRATION IN RELATION TO POSTTRACHEOSTOMY INFECTION

\begin{tabular}{|c|c|c|c|}
\hline & $\begin{array}{c}\text { Number } \\
\text { in } \\
\text { Group }\end{array}$ & $\begin{array}{l}\text { Post- } \\
\text { operative } \\
\text { Infection }\end{array}$ & $\underset{\text { Infection }}{\text { No }}$ \\
\hline $\begin{array}{l}\text { Previous antibiotic administra- } \\
\text { tion for any reason .. } \\
\text { No previous antibiotic admin- } \\
\text { istration } \\
\text { Prophylactic administration of } \\
\text { antibiotic in the absence of } \\
\text { infection } \quad . . \quad \ldots\end{array}$ & $\begin{array}{l}9 \\
9\end{array}$ & $\begin{array}{l}8 \\
8 \\
2\end{array}$ & $\begin{array}{l}1 \\
1\end{array}$ \\
\hline
\end{tabular}

received them, appeared to confer some protection.

The incidence of infection is so high that it is difficult to identify causal factors. Previous infection or aspiration of non-infected material appears to predispose to further infection since the only patients who did not develop post-operative infection were free from infection before the tracheostomy was undertaken.

BACTERIOLOGY OF THE RESPIRATORY INFECTION In five patients no evidence of infection was obtained.

Six patients died before the question of posttracheostomy infection could be assessed.

In three further patients with clinical infection severe enough to warrant antibiotic therapy no specimens were submitted for bacteriological examination.

From the remaining 15 patients the following organisms were found in association with clinical evidence of infection: Staphylococcus aureus, 11 ; Proteus species, 4 ; Pseudomonas pyocyanea, 5 ; and other enterobacteriaceae, 9.

\section{DISCUSSION OF SELECTED PATIENTS}

The following four patients were admitted to the Medical Unit under the care of Professor W. Melville Arnott and have been subjected to his personal study. Since they were admitted during 1962, they are not included in the statistical analysis but they illustrate important features of the epidemiology and the danger of secondary infection in the chest following tracheostomy.

CASE 1 This Irish labourer, aged 49, was admitted with acute bronchopneumonia superimposed on chronic bronchitis. He was semiconscious, unable to give a coherent history, and in severe cardiac failure.

A sample of capillary blood analysed by the method of Astrup showed a $\mathrm{Pco}_{2}$ of $65 \mathrm{~mm}$. $\mathrm{Hg}$, a $\mathrm{pH}$ of $7 \cdot 22$, and a base excess of -1 , indicating alveolar hypoventilation with respiratory acidosis. Haemophilus influenzae, sensitive to all antibiotics except penicillin, was grown from an initial sputum specimen. He had already received penicillin and streptomycin which was changed to chloramphenicol. He was uncooperative and unable to cough up sputum. Oxygen was administered in slowly increasing concentrations but his level of consciousness deteriorated and continued to do so in spite of respiratory centre stimulants (nikethamide and diethylamine vanillic acid (Vandid)).

Tracheostomy was done and the patient was ventilated on a Smith-Clarke respirator. The $\mathrm{Pco}_{2}$ fell to $30 \mathrm{~mm}$. $\mathrm{Hg}$ and his clinical condition improved so rapidly that the respirator was discontinued the following day. The tracheostomy tube was removed after 24 hours. A growth of Proteus sp. was obtained from the sputum at the time of removal of the tracheostomy. 
The following day he started to produce a large volume of foul-smelling purulent sputum and his mental state deteriorated. The $\mathrm{Pco}_{2}$ rose to $94 \mathrm{~mm}$. Hg. The tracheostomy tube was re-inserted and I.P.P.R. was given again. Two multiple resistant staphylococci were then recovered from the sputum (phage types 77 and 29/80). These were present in other patients in the main ward. The patient had been nursed in isolation in a separate side ward. The organisms were sensitive to methicillin and erythromycin only. The chloramphenicol was stopped and methicillin started.

Two days later the sputum became green in colour and Pseudomonas pyocyanea sensitive to colomycin was isolated from several specimens. This was associated with clinical deterioration.

The disease ran a stormy course but the patient recovered and was discharged three weeks later.

He illustrates several interesting features. The initial infection was due to $H$. influenzae, an organism commonly pathogenic in patients with chronic bronchitis. Chloramphenicol eliminated $\boldsymbol{H}$. influenzae which was not recovered from subsequent specimens of sputum. The staphylococci were fresh invaders after the tracheostomy had been performed. They were present in several other patients in the main ward, and isolation and barrier nursing in a side ward, but in the same ward area, were ineffective in preventing infection of this patient. The staphylococcal infection was as severe as the initial bronchopneumonia. As the staphylococcal infection was eliminated it was replaced by $P$ s. pyocyanea. This was also associated with clinical manifestations of infection for which treatment was required.

CASE 2 A man, aged 46, had worked in an iron foundry since the age of 14 . He was in receipt of an $80 \%$ disability pension for silicosis and massive pulmonary fibrosis.

On a previous admission 10 specimens of sputum had been examined on successive days. No tubercle bacilli were seen on direct examination of the sputa. Culture and guinea-pig inoculation of the sputa for Myco. tuberculosis were also negative. $H$. influenzae and Neisseria $s p$. were cultured.

He was readmitted to the unit on 13 February 1962. Figure 3 illustrates relevant clinical and bacteriological features of this illness. On admission he was short of breath and had developed atrial flutter; but he was apyrexial. The blood pressure had fallen to $85 / 60$ $\mathrm{mm}$. Hg. Acute cardiac failure was superimposed on his previous respiratory insufficiency. The $\mathrm{Pco}_{2}$ was $62 \mathrm{~mm}$. Hg.

On the day after admission his clinical condition deteriorated and he became distressed and extremely dyspnoeic. In spite of intravenous aminophylline and Vandid he did not improve. Tracheostomy was performed and intermittent positive pressure respiration

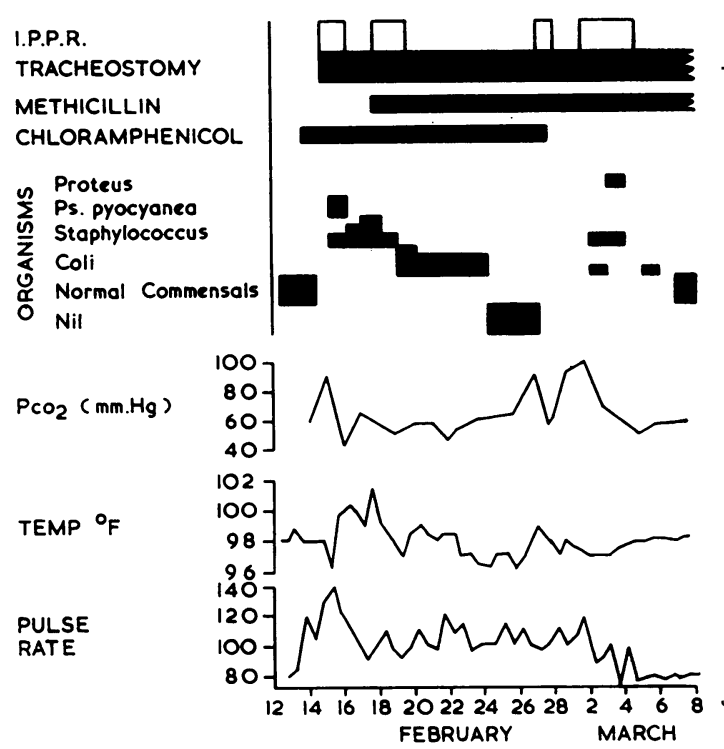

FIG. 3. Data relating to Case 2 during his illness in February and March, 1962.

was given with a Barnet respirator. A culture of his sputum taken shortly before the tracheostomy was performed yielded commensal organisms only.

After tracheostomy he regained consciousness, the atrial flutter reverted to sinus rhythm, and the capillary blood $\mathrm{Pco}_{2}$ fell to $45 \mathrm{~mm}$. $\mathrm{Hg}$. A further specimen of sputum aspirated directly from the trachea produced no growth on culture. He was given chloramphenicol, $500 \mathrm{mg}$. six-hourly. The nursing regime was strict and the patient was isolated.

Twelve hours after tracheostomy had been performed he had improved so much that it was possible to wean him from the respirator.

Seventy-two hours later the patient suddenly deteriorated and developed signs of carbon dioxide narcosis. The tracheostomy tube was re-inserted and I.P.P.R. given again. He had developed acute bronchopneumonia. This aggravated the existing alveolar hypoventilation and led to carbon dioxide retention.

A swab of the tracheostomy wound yielded Staphylococcus aureus (phage type 75/77) sensitive only to erythromycin and methicillin. The same organism was grown from the sputum, and he was given methicillin, $500 \mathrm{mg}$. six-hourly. It may be significant that one of the medical attendants was a nasal carrier of a staphylococcus of the same phage type.

After three days his temperature returned to normal although large quantities of sputum were still being aspirated. The $\mathrm{Pco}_{2}$ fell to $46 \mathrm{~mm}$. $\mathrm{Hg}$. The sputum became foul-smelling, and Escherichia coli was cultured from it. The tracheostomy wound was now septic.

In the subsequent fortnight, the staphylococci disappeared from the sputum. Citrobacter freundii was 
grown from the sputum and the tracheostomy wound, and later Proteus sp. was found in the sputum.

There was a further clinical set-back associated with infection when the $\mathrm{PcO}_{2}$ increased to $92 \mathrm{~mm}$. $\mathrm{Hg}$, but this improved after an hour on the respirator and vigorous physiotherapy.

All antibiotics were discontinued after six weeks, and the patient was discharged to a convalescent home.

We were fortunate to have an opportunity to reassess the patient three months later. He was readmitted following an upper respiratory tract infection. Prior to this he had been able to walk around the house and had been free from purulent sputum.

On this occasion he was apyrexial and the physical signs in the chest had not altered. A culture of sputum produced a heavy growth of pneumococci sensitive to penicillin and tetracycline. Pulmonary function tests showed evidence of airways obstruction.

In the light of his previous history no antibiotics were given. It was felt that these should be reserved for subsequent overt clinical infection of the chest associated with constitutional signs.

This man illustrates several problems. He was a respiratory cripple. The cardiac failure associated with the atrial flutter (and the acute chest infection) precipitated respiratory failure. Tracheostomy saved his life. Subsequent to this he improved, but the tracheostomy wound became infected, and a staphylococcal bronchopneumonia caused dangerous deterioration. The staphylococcus was traced to a nasal carrier on the ward. The infection responded to methicillin. As the staphylococci disappeared, they were replaced in turn by Cit. freundii and Proteus sp. These disappeared without further antibiotic treatment.

This man died six months later. At necropsy the lungs were indurated and fibrotic with multiple apical cavities. The bronchi were fairly clear and the tracheostomy opening had healed with minimal scarring.

CASE 3 A man, aged 27, injured his right thumb and developed tetanus. It was necessary to paralyse him completely with curare, and institute I.P.P.R. to control tetanic spasms. Tracheostomy was performed to facilitate I.P.P.R. Artificial respiration was controlled with frequent $p \mathrm{H}$ and $\mathrm{PcO}_{2}$ estimations. He received penicillin, $\frac{1}{2}$ M.U. b.d., for the control of his infection.

Two days after the tracheostomy had been performed he developed a chest infection.

'Hospital' staphylococci (phage type 80) were cultured from two specimens of sputa. The organisms were sensitive to methicillin and erythromycin. $\mathrm{He}$ was therefore treated with methicillin. The chest infection improved and two days later no organisms could be recovered from the sputum. Four days later his chest infection deteriorated and $E$. coli sensitive to chloramphenicol was recovered from the sputum. Chloramphenicol was added to the antibiotic regime.

Irreversible brain damage occurred following cardiac arrest, and he died a few days later as a result of intractable hypotension.

Necropsy showed marked cerebral softening with bronchopneumonia in both lungs.

Tracheostomy was obligatory since he required prolonged muscular relaxation and I.P.P.R. Prior to the tracheostomy he had no pre-existing chest infection. A high standard of barrier nursing care was maintained. He received prophylactic penicillin therapy both for the local tetanus infection and in order to prevent a respiratory infection. Yet he soon succumbed to a resistant staphylococcus. This was eliminated by methicillin, but once again superinfection with $E$. coli occurred. He died 14 days after admission and, although the chest infection was not the main cause of death, he had a severe bronchopneumonia.

CASE 4 A man, aged 67, with chronic bronchitis had an abdominal aneurysm resected. He had suffered from severe obliterative arterial disease in his legs. The operation was followed by a prolonged period of apnoea which required tracheostomy and I.P.P.R.

A swab taken from the tracheostomy wound on the following morning yielded Streptococcus viridans and a sensitive strain of Staph. aureus (phage type 55/71). In spite of prophylactic chloramphenicol he developed a profuse diarrhoea the following day, and Staph. aureus (phage type 53/77), sensitive only to methicillin and erythromycin, was cultured from the stool. He was therefore given methicillin. The sputum increased in quantity and became purulent. A heavy growth of the same staphylococcus and $E$. coli was obtained. He was also pyrexial and developed clinical signs of the infection on the chest. He improved on methicillin, but $C$ it. freundii appeared in the sputum and this was sensitive only to neomycin and kanamycin. Neomycin and nystatin were added to the regime.

His clinical condition improved, and a smaller volume of sputum was produced, from which only a scanty growth of Staph. aureus and Cit. freundii was isolated. Ps. pyocyanea was later cultured from the sputum but was not associated with fresh symptoms, and no further antibiotic therapy was given. He was discharged one month after operation.

The patient had no chest infection when tracheostomy was undertaken. He developed a staphylococcal enteritis with organisms present in other patients who were in the ward at the time. This occurred in spite of strict barrier nursing in an isolation cubicle, and chloramphenicol prophylaxis. Staphylococcal pneumonia ensued; when this subsided on methicillin, Cit. freundii, coliforms, and later Ps. pyocyanea colonized the sputum, illustrating how readily these organisms proliferate 
when the normal bacterial flora is removed by antibiotics.

\section{DISCUSSION}

REVIEW OF THE LITERATURE There are many problems in the management of a tracheostomy; most of these have already been discussed very adequately in the existing literature. They include mechanical factors, the problem of crusting of tracheal secretions, and the techniques to be used for aspiration (Negus, 1961 ; Crawford, 1961). Little attention has been paid to respiratory infection after tracheostomy. Nelson and Bowers (1957) reviewed 310 cases of tracheostomy but did not mention pulmonary infection as a complication. Other large series mention infection, but the incidence and bacteriological characteristics of the infection received no attention. Atkins (1960) reviewed 526 cases, and a table in his paper revealed that 129 of these cases came to necropsy. Pneumonia was found in $99(76 \%)$ but there is no comment upon this in the text. Head (1961), in another exhaustive series of patients from the Massachussets General Hospital, described infection as a late complication in 80 out of 462 cases ; the nature of the infection was not classified and any manifestations from superficial sepsis to pulmonary infection were included in these figures. The July number of Anesthesiology (1962) is devoted to a symposium on inhalation therapy, but while tracheostomy and I.P.P.R. are discussed in detail, infectious complications are not considered. By contrast Lepper, Kofman, Blatt, Dowling, and Jackson (1954) described the bacteriological findings in 72 poliomyelitis cases where respiratory paralysis was treated with tracheostomy and I.P.P.R. Pulmonary infection occurred in every case and the causative organisms were wide but included Staph. pyogenes, Pseudomonas sp., and Proteus sp. It is not clear whether the infected cases represented all the poliomyelitis cases with tracheostomy in one hospital, or only those who developed respiratory infection, but the evidence for repeated infection with change in the organism responsible is formidable and illustrates the ease with which infection occurs in these patients.

In two other small but intensively studied series (Davidson, 1959 ; Nisbet, and Wilson, 1958) the bacteriology of infection is discussed since respiratory infection was the indication for tracheostomy; the incidence of post-operative respiratory infection is difficult to assess. Smythe and Bull (1961) and Cole (1959) found that some patients who had tetanus and who were treated by tracheostomy and
I.P.P.R. developed a fatal pneumonia: but they give no bacteriological data.

In an account of the management of tracheostomy, Frew (1961) makes the point that tracheostomy patients are readily cross-infected with organisms in the hospital environment and that nursing of tracheostomy patients in individual cubicles should lead to a reduction in the incidence of infection. He gives no illustrative cases and no specific information on the causal organisms.

INCIDENCE OF INFECTION Our experience is that cross infection with hospital pathogenic organisms is almost certain to occur within a week of tracheostomy being established. It is in accord with the view that an abnormal respiratory tract is readily colonized by resistant staphylococci in hospitals as illustrated for bronchitics by Mitchell, Dunn, Lees, and Hedges (1961). Other organisms causing infection were also well recognized crossinfecting types such as Ps. pyocyanea and Proteus $s p$.

FACTORS IN CAUSATION OF INFECTION The problem is to decide whether the tracheostomy can be incriminated as the cause of the patient's infection. Many patients in our series had tracheostomy performed for conditions in which respiratory tract infection was already established or its development inevitable. Aspiration of material and failure to clear secretions from the respiratory tract occurred because these patients were unable to cough. Nelson has stressed the importance of this syndrome of 'secretional ventilatory obstruction".

In two patients, who died shortly after tracheostomy had been performed, and in whom the period of survival was too short to assess the role of respiratory infection, the trachea and bronchi were congested and filled with mucopurulent secretion. There were numerous areas of atelectasis and in some parts frank infection. It could be argued that infection is the inevitable result of such aspiration and that this factor is the indication for tracheostomy. The effective protective mechanisms of the respiratory tract are lost and colonization may occur with organisms that are not pathogenic as primary invaders. Under these conditions the tracheostomy cannot be blamed for the infection but it appears probable that the type of infection is influenced by the presence of a tracheostomy. Bronchopneumonia and aspiration pneumonia in hospitals are frequently complicated by staphylococcal infection, but other organisms such as $\boldsymbol{H}$. influenzae and Strep. pneumoniae are also common causal agents. 
In our series not a single case was infected with these latter organisms while on tracheostomy. In addition in a number of cases bacteriological data showed that one causal organism was present in the infected sputum at the time tracheostomy was performed. This was subsequently replaced by Staph. aureus, Ps. pyocyanea, or other species in 15 patients who lived for three days or more.

Most patients with a tracheostomy received routine antibiotic cover. The majority of staphylococcal infections were with organisms resistant to penicillin, streptomycin, and tetracycline, and in many units these are the most popular 'prophylactic' antibiotics. In this hospital the majority of staphylococci isolated were only sensitive to erythromycin, chloramphenicol, and methicillin. On the Professorial Medical Unit chloramphenicol-resistant staphylococci were also common (Fig. 4). Thus to protect in-patients from staphylococcal

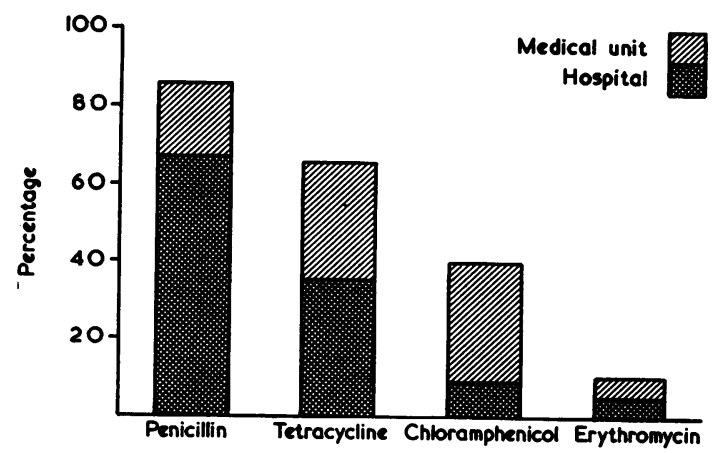

FIG. 4. Percentage of strains of Staph. aureus isolated in Queen Elizabeth Hospital at large and in the Professorial Medical Unit resistant to various antibiotics.

infection with a prophylactic antibiotic one of these latter antibiotics should be chosen in preference to the former: the problem of antibiotic prophylaxis however is not as simple as this. In patients who have an established staphylococcal infection, treatment with an effective antibiotic was often followed by the establishment of a further resistant infection usually caused by $P$ s. pyocyanea.

There is usually no question about the pathogenicity of Staph. aureus in pulmonary conditions, as measured by the volume of purulent sputum, the pulmonary changes, and the constitutional symptoms produced in the patient. In the case of replacement infection with Gram-negative bacteria following successful anti-staphylococcal therapy the association is not so clear. Often there is much less constitutional disturbance, and some of our cases illustrate the difference.
In the normal person inspired air passes through the upper respiratory tract where it is warmed and filtered so effectively that swabs from the main bronchi taken through a bronchoscope are sterile (Brumfitt, Willoughby, and Bromley, 1957). The tracheostomy bypasses the upper respiratory tract. Most respirators include a humidifier and to some it is possible to fit an air filter. Even when air is filtered there are periods when the patient is disconnected from the respirator and respires directly through his tracheostomy. Thus airborne infection of the bronchial tree may be readily and directly achieved. The catheter used for suction is another means by which infection may be acquired. The tracheostomy wound may also represent a portal of entry for bacteria.

Patients who have a tracheostomy cannot cough effectively. An efficient cough requires a closed glottis, which allows the development of momentary, high intra-thoracic pressure. The glottis is opened and an explosive current of air at high velocity is directed up the trachea. The velocity of the blast is important since this determines the effectiveness of the cough. This prevents the tracheostomy patient from clearing his own trachea and bronchi effectively since the cough is shallow. If the patient can cough, he does not produce sputum in the pharynx but often expectorates it through the tracheostomy opening. This is unhygienic and unpleasant; it soils the wound and the tracheostomy dressings, and is a way of disseminating and spraying the organisms in the ward.

MANAGEMENT The epidemiology of the infection probably differs very little from that of wound infection. In one respect it is similar to a burn. An exposed surface (the bronchial and tracheal mucosa) is covered with dead material and this probably accounts for the patient's increased susceptibility to infection.

In the management of tracheostomy there are two main problems in relation to infection; the patient must be protected from infection but if infection occurs other patients must be prevented from acquiring it. Both these objectives demand the isolation of the patient.

In our series many of these patients were isolated in single rooms within the ward area and barrier nursing was attempted but could not always be enforced. In spite of isolation patients become infected with organisms present in the ward at the time of their isolation. This implies that for isolation to be successful the patient should be nursed in a true isolation unit unconnected with general hospital wards. Carriers of 
dangerous organisms should be rigorously excluded from the isolation room. The question of visitors is also difficult ; they usually do not carry dangerous organisms but, if permitted to enter, they must adopt the same precautions as the staff. Attention must be paid to the hygiene of the tracheostome, and for this purpose disposable sterile catheters are the most suitable for tracheal aspiration. It is important to be gentle with suction which should never be applied while inserting the catheter. The air supply for respiration in humidifiers should be bacteria-free, since any inspired bacteria will be implanted directly into the lower respiratory tract. If antibiotics are to be administered they should be appropriate to combat likely infecting organisms; other antibiotics may actually favour such infection. Daily swabs of the tracheal aspirate should be submitted for bacteriology. In this way any change in flora can be recognized and combated at the earliest possible moment if clinically indicated.

Tracheostomy is such an effective procedure in the management of many conditions that would otherwise prove fatal that it is essential for it to be managed efficiently.

Pulmonary infection is a dangerous hazard and counteracts many of the benefits of tracheostomy. The main purpose of performing the tracheostomy is to reduce and remove the retained secretions and reduce the amount of work performed by the lungs for a given amount of gas exchange. Pulmonary infection aggravates this problem and undoes the benefits which would accrue from the tracheostomy.

\section{SUMMARY}

Twenty-nine patients submitted to tracheostomy in the Queen Elizabeth Hospital in 1961 have been examined for evidence of pulmonary infection.

Twenty-three lived for more than three days and, of these, 18 became infected following tracheostomy. Eleven infections were due to antibioticresistant staphylococci and five to Ps. pyocyanea.
The nature of the infecting organisms clearly indicated the hospital environment as the source of the infection.

Factors influencing the establishment of infection have been discussed and some recommendations made about the management of tracheostomy patients.

We are indebted to Professor W. Melville Arnott for permission to study the patients on his unit and for his criticism and advice, and to Professor A. L. d'Abreu for encouragement and suggestions.

\section{REFERENCES}

Anesthesiology (1962). Symposium-Inhalational Therapy. 23, pp. 407 575.

Atkins, J. P. (1960). Current utilization of tracheostcmy as a therapeutic measure. Laryngoscope (St. Louis), 70, 1672.

Brumfitt, W., Willoughby, M. L. N., and Bromley, L. L. (1957). An evaluation of sputum examination in chronic bronchitis. Lancet, ii, 1306.

Bryce-Smith, R. (1950). The management of head injuries from an anaesthetist's point of view. Brit. med. J., $2,322$.

Cawthorne, T., Hewlett, A. B., and Ranger, D. (1959). Tracheostomy in a respiratory unit in a neurological hospital. Proc. roy. Soc. Med., $52,403$.

Cole, L. B. (1959). Tracheostomy in tetanus. Ibid., 52, 411.

Crawford, O. B. (1961). The anesthesiologist's responsibilities in tracheostomy. Anesthesiology, 22, 86.

Davidson, L. A. G. (1959). Tracheotomy in acute respiratory disease. Lancet, 1, 597.

Frew, I. J. C. (1961). Tracheostomy and controlled respiration. J. Laryng., 75, 136

Galloway, T. C. (1943). Tracheotomy in bulbar poliomyelitis. $J$ Amer. med. Ass., 123, 1096

Head, J. M. (1961). Tracheostomy in the management of respiratory problems. New Engl. J. Med., 264, 587.

Hugh-Jones, P. (1958). Oligopnoea. Proc. roy. Soc. Med., 51, 104.

Lassen, H. C. A. (1953). The 1952 epidemic of poliomyelitis in Copenhagen, with special reference to the treatment of acute respiratory insufficiency. Lancet, $i, 37$.

Lepper, M. H., Kofman, S., Blatt, N., Dowling, H. F., and Jackson, G. G. (1954). Effect of eight antibiotics used singly and in combination on the tracheal flora following tracheotomy in poliomyelitis. Antibiot. Chemother., 4, 829.

Maciver, I. N., Frew, I. J. C., and Matheson, J. G. (1958). The role of respiratory insufficiency in the mortality of severe head injuries. of respiratory insufficiency in the mortality of severe head injuries.

Meade, J. W. (1961). Tracheotomy-its complications and their management. New Engl. J. Med., 265, 519.

Mitchell, A. A. B., Dunn, R. I. S., Lees, T. W., and Hedges, C. K. (1961). Staphylococcal pulmonary infection. Lancet, ii, 669.

Negus, V. (1961). Protection of the respiratory tract. Brit. med. J., $2,723$.

Nelson, T. G. (1958). Tracheotomv: A Clinical and Experimental Studv. Williams and Wilkins, Baltimore.

Nelson, T. G., and Bowers, W. F.(1957). Tracheotomy-indications, advantages, techniques, complications, and results. J. Amer. med. Ass., 164, 1530 .

Nisbet, H. I. Armstrong, and Wilson, F. (1958). The treatment of acute respiratory infection in infants. Brit. $J$. Anaesth, $30,419$.

Smythe, P. M., and Bull, A. B. (1961). Treatment of tetanus with $\sigma$ special reference to tracheotomy. Brit. med. J., 2, 732. 